Itinéraires Itinéraires

Littérature, textes, cultures

2020-1| 2020

Les cultures du chapitre

\title{
Finir in medias res : chapitrage et division en actes
}

In Medias Res Endings: Chapitering and Act-Division

\section{Marc Douguet}

\section{OpenEdition}

Journals

Édition électronique

URL : http://journals.openedition.org/itineraires/7291

DOI : 10.4000/itineraires. 7291

ISSN : 2427-920X

Éditeur

Pléiade

Référence électronique

Marc Douguet, «Finir in medias res : chapitrage et division en actes », Itinéraires [En ligne], 2020-1

2020, mis en ligne le 02 octobre 2020, consulté le 13 novembre 2020. URL : http://

journals.openedition.org/itineraires/7291 ; DOI : https://doi.org/10.4000/itineraires.7291

Ce document a été généré automatiquement le 13 novembre 2020.

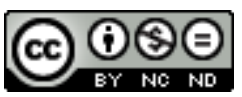

Itinéraires est mis à disposition selon les termes de la licence Creative Commons Attribution - Pas d'Utilisation Commerciale - Pas de Modification 4.0 International. 


\title{
Finir in medias res : chapitrage et division en actes
}

\author{
In Medias Res Endings: Chapitering and Act-Division
}

Marc Douguet

1 La division d'un roman en chapitres et la division d'une pièce de théâtre en actes reposent sur une même dialectique de la coupure et de la jointure, de la rupture et de la liaison, de la discontinuité et de la continuité. Philippe Hamon (2017: 7) englobe ces deux phénomènes dans l'ensemble plus large des modes de structuration des œuvres :

Le cadre d'un tableau, l'entracte et les divisions en scènes et actes au théâtre, la pause entre les mouvements dans une pièce musicale, le blanc entre les strophes et aux marges extérieures d'un poème, la ponctuation et les alinéas d'une page imprimée, les chapitres d'un roman avec leur numérotation, leurs titres ou leurs sous-titres, les enchâssements d'un récit, les refrains récurrents d'une chanson, la tomaison d'une œuvre, les plans dans un film [...] tout cela fait partie de la question : où, comment, et pourquoi couper, faut-il couper ou faut-il coudre?

Ugo Dionne (2008: 515) consacre pour sa part un développement à «l'influence du théâtre sur le genre romanesque » et montre comment «les deux grandes divisions théâtrales, l'acte et la scène, fournissent des exemples de structuration littéraire qu'on a tenté d'appliquer à la narration en prose ».

2 Il s'agira ici de contribuer à l'étude comparée de ces deux dispositifs et, malgré la différence entre leurs modes de mimésis respectifs, de s'interroger sur ce qu'ils partagent. Plus précisément, on cherchera à savoir si l'effet produit sur le spectateur par la division en actes peut être pensé dans les mêmes termes que celui que produit sur le lecteur la division en chapitres. On espère ainsi, par cette mise en parallèle, pouvoir mieux cerner le fonctionnement de l'un comme de l'autre.

3 Les questions soulevées par le chapitrage et la division en actes sont beaucoup trop nombreuses pour être toutes prises en compte ici. Dans la lignée de la remarque de Philippe Hamon, on verra avant tout dans le chapitrage et la division en actes des procédés de segmentation: on s'intéressera donc moins aux chapitres et aux actes en 
tant qu'unités constituantes qu'aux blancs qui les séparent, et au phénomène de discontinuité qui va de pair avec ces dispositifs.

\section{Continuité ou discontinuité : un débat théorique commun}

4 Un même débat théorique, récurrent depuis l'Antiquité, semble toucher les deux genres. Un certain nombre d'auteurs ou de critiques insistent en effet sur les risques que présentent la discontinuité du spectacle aussi bien que celle du roman. Ainsi, le principe même de la segmentation du texte narratif a parfois été remis en cause. Dans l'avertissement d'Agathonphile ou les Martyrs siciliens, Jean-Pierre Camus se justifie de ne pas avoir divisé son roman en chapitres :

Que si je n'ai point fait de sommaire à ce livre, ni ne l'ai point distingué en chapitres, c'est parce qu'il me semble que le raccourcissement de ceux-là fait trouver ennuyeuse la nécessaire amplification du discours, et les coupures de ceuxci brisent la douceur de la tissure juste et égale. [...] Outre que c'est fomenter la paresse, cela ôte cette gracieuse suspension d'esprit en laquelle consiste toute la délectation de la lecture d'une histoire ${ }^{1}$. (Camus 1662 : non paginé)

Comme le souligne Barbara Selmeci Castioni (2011 : 173-174), cet exemple illustre bien les réticences plus générales de l'âge classique à l'égard du chapitrage : "La mise en texte continue à laquelle se rattachent nombre de romans à cette époque peut être comprise comme relevant d'une volonté de renforcer l'emprise fictionnelle du récit romanesque. » Tout est fait pour que le lecteur se plonge entièrement dans le roman et oublie la matérialité de l'ouvrage qu'il tient entre ses mains : le changement de chapitre est vu, au même titre que l'entracte théâtral, comme une rupture de l'illusion mimétique et un dommageable retour à la réalité.

5 L'argumentation de Camus est d'autant plus intéressante que celui-ci emploie le terme de «suspension » en lui donnant un sens bien différent de celui auquel nous sommes habitués. Dès le XVII siècle, la "suspension ", nous y reviendrons, correspond à peu près au «suspense ", et c'est précisément un effet auquel contribue fortement la segmentation du texte : le terme s'applique alors au récit ou au spectacle qui, suspendu avant son terme, suscite notre attente. Or ici, c'est au contraire la continuité qui crée la "suspension", et c'est l'esprit du lecteur lui-même qui se trouve "suspendu", abdiquant pour un temps toute distance critique pour n'être qu'un pur témoin des événements relatés. Camus (1662: n. p.) utilise d'ailleurs l'expression «suspendre son jugement » dans la phrase qui précède l'extrait cité : «Au moins te supplié-je, ayant lu cette histoire, de suspendre ton jugement jusques à ce que tu aies lu les justifications de cet éloge. »). Ce discours se retrouve dans la théorie dramatique, avec deux arguments principaux. D'une part - et l'enjeu est ici spécifique au théâtre - on craint de voir le public quitter la salle à l'occasion de l'entracte.

6 Celui-ci peut le faire volontairement. Donat avance cette raison pour expliquer que les comiques latins n'aient pas divisé leurs pièces en actes :

Les actes y sont particulièrement imbriqués et difficiles à séparer si l'on n'est pas tant soit peu spécialiste, parce que, pour tenir en haleine le spectateur, notre poète veut que les cinq actes n'en fassent pour ainsi dire qu'un, afin qu'il n'ait pas le temps de respirer et que, devant un enchaînement d'événements qui se succèdent avec de rares pauses ici ou là, le spectateur fatigué ne quitte pas le théâtre avant le lever de rideau ${ }^{2}$. 
7 Pour défendre le dispositif de la tragédie antique (où les chants du chœur rythment le spectacle sans l'interrompre) contre la division en actes pratiquée au XVII siècle (où un simple intermède de violon, sans lien avec l'action, introduit une discontinuité beaucoup plus forte), Dacier évoque quant à lui le risque d'un départ involontaire, le spectateur croyant sincèrement que le spectacle est fini :

Mais par où le premier acte tient-il au second, le second au troisième, etc. quand une chose aussi étrangère que les airs de violon les a séparés, et qu'est-ce qui me porte à demeurer dans la même place en attendant que les acteurs qui sont rentrés reviennent? Qui m'a dit qu'ils reviendront? Oh! Mais ils reviennent toujours, c'est la coutume. Plaisante sûreté ! Mais celui qui voit la tragédie pour la première fois, est-il instruit de cette coutume ? Il faut donc qu'il l'apprenne de son voisin, et que ce voisin lui en réponde. (Dacier 1692 : 314-315)

8 D'autre part, la théorie dramatique rejoint parfois le discours sur le roman en critiquant la rupture de l'illusion mimétique induite par les entractes. Comme Dacier, Jean-Marie Clément est un défenseur du dispositif grec antique :

Les chœurs des anciens avaient cet avantage particulier qu'en liant toutes les parties du spectacle et ne quittant la scène qu'en des occasions indispensables, ils fixaient rigoureusement l'unité du lieu et en même temps entretenaient l'émotion, attachaient l'esprit du spectateur et l'empêchaient de prêter trop attention à la durée fictive des événements. Nos froids entractes produisent un effet tout contraire; ils nous invitent à la réflexion; leurs intervalles réguliers nous rappellent à ces intervalles dont nous mesurons le temps, et qu'il fallait nous faire oublier. (Clément $1784:$ 38-39)

9 On retrouve là les mêmes arguments que chez Camus: la continuité du spectacle « attache » l'esprit, tandis que les entractes laissent le jugement libre et « invitent à la réflexion ». Cependant, l'adhésion du spectateur est ici recherchée dans une finalité bien précise : il s'agit avant tout de garantir la vraisemblance temporelle du spectacle en faisant en sorte que le spectateur ne se rende pas compte de l'écart entre la « durée fictive des événements » et la durée réelle du spectacle qui les représente. Les entractes peuvent fonctionner comme des ellipses, mais celles-ci doivent rester discrètes.

10 Très tôt, d'autres théoriciens ont au contraire insisté sur le fait que la division en actes aussi bien que celle en chapitres permettaient à l'esprit du spectateur de se reposer, et qu'elles étaient à ce titre nécessaires.

11 On connaît la célèbre image de l'interchapitre comme "auberge" proposée par Fielding ([1742] 1964: 83-84). Ugo Dionne donne également de nombreux exemples d'une semblable justification dans des paratextes ${ }^{3}$. Cet argument est tout autant un lieu commun de la théorie dramatique. Pour Charles Estienne, la nécessité de permettre au spectateur de se reposer est à l'origine de la division en actes : l'acte « fut inventé pour ne détenir trop longuement les auditeurs en une même chose et pour recréer les esprits par intervalles » (Estienne [1542] 1949 : 38-39). D'Aubignac, après bien d'autres, reprend la même idée en la développant plus longuement :

L'expérience nous apprend que les hommes n'ont point d'attention assez forte pour supporter une pièce de théâtre toute entière et sans aucun relâche, vu qu'un seul acte nous est ennuyeux et insupportable quand il est un peu trop long ; tant l'esprit humain est peu capable de s'attacher longtemps et attentivement à un seul objet ! (D’Aubignac [1657] $2001: 350$ )

12 Le topos du repos du spectateur se développe donc progressivement sous la forme d'une considération psychologique et anthropologique sur nos capacités d'attention et de concentration. L'objectif est finalement le même que celui des détracteurs de la 
segmentation (il faut que le spectateur «s'attache » au spectacle), mais les théoriciens prennent ici acte, avec un certain pragmatisme, des limites de l'esprit humain.

Le repos du spectateur n'est pas le seul argument avancé par les défenseurs de l'entracte. Même si l'idée est beaucoup moins développée, les théoriciens y voient aussi une occasion pour le spectateur de participer activement au spectacle en émettant un jugement sur ce qu'il vient de voir. Ainsi, pour Corneille ([1660] 1999: 140-141), les intermèdes de violons permettent, contrairement aux chants du chœur, d'offrir une véritable coupure, ni trop longue, ni courte : «L'esprit de l'auditeur se relâche durant qu'ils jouent, et réfléchit même sur ce qu'il a vu pour le louer ou le blâmer, suivant qu'il lui a plu ou déplu4.» Cette idée se retrouve d'ailleurs dans le texte de Fielding ([1742] 1964: 84) auquel nous faisions allusion plus haut : «Le voyageur s'arrête quelque temps pour se reposer et réfléchir à ce qu'il a vu dans les régions qu'il a déjà traversées ${ }^{5}$. » Il est frappant de voir à quel point cette conception s'oppose, presque mot pour mot, à celle de Camus ou Clément, qui reprochaient justement aux entractes ce dont Corneille et Fielding les louent : « Ils nous invitent à la réflexion. »

À côté de ces raisons positives d'introduire des entractes, les théoriciens ont également réfléchi aux procédés à mettre en œuvre pour contrebalancer les effets néfastes de la discontinuité, qui menace l'unité du spectacle. La question est toujours celle que pose la fiction théorique du spectateur naïf imaginée par Dacier : pourquoi savons-nous que le spectacle est seulement interrompu et non pas fini ? pourquoi attendons-nous la suite ?

C'est ici que l'on retrouve la notion de «suspense », ou, dans la langue de l'époque, de "suspension ». Corneille y a longuement réfléchi :

Il n'y doit avoir qu'une action complète, qui laisse l'esprit de l'auditeur dans le calme, mais elle ne peut le devenir que par plusieurs autres imparfaites, qui lui servent d'acheminements et tiennent cet auditeur dans une agréable suspension. C'est ce qu'il faut pratiquer à la fin de chaque acte pour rendre l'action continue. Il n'est pas besoin qu'on sache précisément tout ce que font les acteurs durant les intervalles qui les séparent ni même qu'ils agissent lorsqu'ils ne paraissent point sur le théâtre, mais il est nécessaire que chaque acte laisse une attente de quelque chose qui se doive faire dans celui qui le suit. Si vous me demandiez ce que fait Cléopâtre dans Rodogune, depuis qu'elle a quitté ses deux fils au second acte jusqu'à ce qu'elle rejoigne Antiochus au quatrième, je serais bien empêché à vous le dire, et je ne crois pas être obligé à en rendre compte. Mais la fin de ce second prépare à voir un effort de l'amitié des deux frères pour régner, et dérober Rodogune à la haine envenimée de leur mère. (Corneille [1660] 1999 : 134)

Ce qui garantit la continuité de l'action par-delà les entractes, c'est justement qu'elle n'est achevée, "complète " qu'au moment du dénouement. Jusqu'à ce point, son incomplétude empêche le spectateur de considérer que la pièce est finie.

\section{Sortie des personnages et rideau de scène : quel critère de structuration pour le spectacle?}

Si la division en actes peut accroître le suspense en interrompant le spectacle à un moment où la situation, instable, fait attendre une suite, nous sommes cependant bien loin de l'effet - largement étudié - que peut susciter une fin de chapitre. Très souvent cité, le récit du combat de Don Quichotte contre le Biscayen peut être vu comme l'exemple type du cliffhanger (notamment dans la mesure où les commentaires 
métanarratifs qui l'accompagnent insistent avec ironie sur le procédé). La fin de la première partie se termine sur une situation paroxystique :

Don Quichotte s'en venait, comme on l'a dit, contre le prudent Biscayen, l'épée haute et décidé à le fendre en deux, et le Biscayen l'attendait tout de même, l'épée en l'air et abrité sous son coussin, et tous les assistants, épouvantés, étaient suspendus à ce qui allait advenir des grands coups dont ils se menaçaient. (Cervantès [1605] $2001:$ :456)

Cervantès joue avec les codes de la narration en interrompant une action en plein cours, de manière abrupte et soudaine. Wolfgang Iser ([1973] 1985 : 332) a bien montré d'une part que ce procédé repose sur une information volontairement soustraite au lecteur, et, d'autre part, qu'il a pour conséquence d'accroître la participation de ce dernier au récit :

Le plus souvent, le récit est interrompu au moment où est créée une tension qui appelle une résolution pressante, ou bien au moment précis où l'on aurait voulu connaître l'issue des événements que l'on vient de lire. [...] Un tel effet de suspense fait que nous cherchons à nous représenter immédiatement l'information qui nous manque sur la suite des événements. Comment la situation va-t-elle évoluer? Plus nous nous posons ce genre de question, et plus nous participons au déroulement des événements.

Dans d'autres cas, la coupure interchapitrale survient immédiatement après une révélation, une découverte qui participe également à la tension dramatique, mais suscite moins un effet de suspense que de surprise ${ }^{6}$. C'est ainsi que, dans Je m'en vais, Delahaye réapparaît à la toute fin du chapitre 33, alors qu'on le croyait mort :

S'arrêtant à deux mètres de Baumgartner, il parut hésiter un instant puis s'approcha de lui. Excusez-moi, dit-il en posant légèrement deux doigts sur l'épaule de cet homme, qui se retourna.

Tiens, dit Ferrer. Delahaye. Je me disais bien, aussi. (Échenoz 1999 : 227)

Cette fin de chapitre nous frustre tout autant d'une information, quoique d'une autre manière : ce qui nous manque, c'est d'une part une explication (comment se fait-il que Delahaye soit toujours en vie ?), et, d'autre part, la suite immédiate de son dialogue avec Ferrer, dont nous n'avons que le début.

Ces deux exemples correspondent à ce que Philippe Hamon (1975: 509) a théorisé sous le nom de " clausule ouvrante ", qui déclenche " une activité prospective d'attente chez le lecteur ». Celle-ci ne crée pas à elle seule l'effet de suspense ou de surprise, qui est inhérent à la situation, mais suscite, selon l'expression de Randa Sabry (1992 : 174), un " effet de rupture », qui exacerbe la tension en interrompant brutalement le récit juste après une péripétie ${ }^{7}$.

Or à l'âge classique, le découpage en actes ne permet pas de parvenir à un résultat comparable, car il repose entièrement sur les mouvements mêmes des personnages, et non sur un rideau qui permettrait de cacher le plateau. En France, deux modèles se succèdent.

Dans le premier modèle, majoritaire jusque dans les années 1640 , l'entracte doit coïncider avec un moment où le plateau est vide, c'est-à-dire où l'ensemble des personnages est sorti (certains pouvant par ailleurs revenir dès le début de l'acte suivant). La réciproque n'est pas vraie: le plateau peut être momentanément vide à l'intérieur d'un acte, quand deux scènes successives ne sont pas "liées ", et n'ont aucun personnage en commun. Ces ruptures de la liaison des scènes introduisent donc un niveau de structuration intermédiaire entre la scène et l'acte. Au contraire, dans le second modèle, qui s'impose à partir des années 1640 , non seulement le plateau doit 
être vide au moment de l'entracte, mais il ne peut l'être qu'à ce moment. Chaque acte forme un bloc cohérent à l'intérieur duquel les personnages présents sur le plateau se renouvellent, mais progressivement, par tuilage ${ }^{8}$.

Dans tous les cas, la division en actes diffère de la division en chapitres en ce qu'elle repose sur un critère fixe, qui appartient à la diégèse. Le dispositif est intrinsèquement lié au contenu de l'œuvre: on peut appliquer au théâtre la notion de «segmentation immanente » proposée par Ugo Dionne (2008:250), par laquelle c'est «le texte luimême qui, par divers moyens, prend en charge quelques-unes des fonctions de gestion et d'organisation que remplit autrement le dispositif». Pour modifier la division en actes, il faut nécessairement intervenir sur les mouvements d'entrée et de sortie des personnages.

21 Au contraire, le chapitrage peut être ajouté une fois terminée la phase d'écriture, voire n'être qu'une intervention éditoriale, voire être introduit sans concertation avec l'auteur. C'est aussi ce qui rend son analyse aussi complexe, car, comme le rappelle Aude Leblond (2012), il ne repose pas sur un critère unique, objectif et invariant, ni à l'échelle du genre dans son ensemble, ni même à l'échelle d'une seule œuvre :

La disposition romanesque semble trop lâche, trop floue ou trop différente d'un roman à l'autre pour être perçue comme un élément véritablement signifiant. [...] Il paraît impossible de formuler la loi du chapitrage des Trois Mousquetaires, qui ne présente ni symétrie ni schéma d'ensemble.

La fixité et l'invariance du découpage en actes ont au contraire pour corrélat qu'il est possible de le reconstituer même en l'absence de marques éditoriales: il suffit de repérer les moments où le plateau est vide. C'est à cet exercice que se sont livrés les premiers éditeurs du théâtre de Térence, qui se trouvaient face à des manuscrits sans aucune division :

Mais sur quels critères et comment il est possible (bien que ce soit difficile) de la comprendre [la division en actes] et de la repérer, cela vaut la peine de l'apprendre.

[...] Il faut donc observer attentivement où et quand la scène se vide de tous les personnages, en sorte que l'on peut y écouter le chœur ou le flûtiste. Quand nous voyons cela, nous devons reconnaître qu'il y a là la fin d'un acte?

Comme le souligne Donat, cette opération reste difficile et sujette à l'interprétation. Elle l'est d'autant plus dans le cas de Térence que les premiers éditeurs ont cherché à tout prix à appliquer le précepte horatien de la division en cinq actes à des textes qui lui sont antérieurs et qui reposaient sur une action continue ${ }^{10}$. Mais l'important est que cette entreprise soit, dans l'absolu, possible : si interprétation il y a, elle porte sur la diégèse même (tel personnage sort-il du plateau ou reste-t-il présent?) et non directement sur le dispositif. Certes, le premier modèle du découpage en actes laisse une certaine marge de liberté dans la mesure où l'on peut faire sortir du plateau la totalité des personnages à l'intérieur d'un acte. Néanmoins, le dispositif théâtral reste toujours beaucoup plus contraint que le dispositif romanesque.

La nature particulière de ce dispositif a une conséquence fondamentale sur l'effet qu'il produit. Dans le roman, l'effet de rupture que nous avons pu observer vient d'une réticence d'information: comme s'il nous raccrochait au nez, le récit s'interrompt au lieu de nous délivrer l'information souhaitée. Or cette interruption est le fait du narrateur.

Dans le théâtre de l'âge classique, ce sont les personnages eux-mêmes, qui, en sortant, prennent en charge le dispositif. Il devient dès lors a priori impossible de couper une action en plein milieu. Même si, comme le préconise Corneille, la dernière scène de 
l'acte peut susciter un fort effet d'attente à l'échelle de la pièce, l'entracte survient toujours, à l'échelle de la scène elle-même, sur un temps faible: le moment où le dialogue s'achève, où les personnages se séparent ou sortent.

Ainsi, à la dernière scène de l'acte III du Cid, Don Diègue annonce à Rodrigue l'arrivée des Maures, et Rodrigue sort pour aller combattre. Mais la scène se termine sur ces mots (Corneille 1637 : III, 6, v. 1109-1110) : « Viens, suis-moi, va combattre, et montrer à ton roi / Que ce qu'il perd au comte il le recouvre en toi. » La coupure suscite un suspense à moyen terme (Rodrigue triomphera-t-il ?). Mais à court terme, la situation est stable. Le spectateur n'est donc pas frustré par la rétention d'une information qui lui aurait été délivrée si l'entracte n'avait pas eu lieu: l'entracte interrompt la représentation, mais, dans la mesure où il suppose que le plateau soit vide, il n'y a de toute façon plus rien à représenter au moment où il survient. L'acte se finit sur une cadence mineure, la sortie des personnages préparant l'interruption du spectacle à proprement parler. La plupart du temps, le mouvement de sortie est même thématisé dans le discours des personnages, qui disent où ils vont, ou du moins annoncent leur départ - ce qui ne fait qu'adoucir encore plus la rupture que constitue l'entracte. Le verbe «aller » y est d'ailleurs récurrent. La fin de l'acte II de Rodogune, que Corneille prenait comme exemple d'" agréable suspension ", prépare effectivement "à voir un effort de l'amitié des deux frères » (Corneille [1660] 1999: 134), mais il faut du temps pour que celui-ci se réalise. Dans l'immédiat, l'entracte n'interrompt pas une action en cours, et la scène se clôt sur le départ des personnages (Corneille 1647: II, 4, v. 757-758) : «Allons, et soyez sûr que même le trépas / Ne peut rompre des nœuds que l'amour ne rompt pas. »

D’Aubignac défend même une conception de l'entracte qui nie toute interruption du spectacle. Selon lui, une fois défini le lieu que représente le plateau, il est impossible d'y supposer qu'une action s'y déroule sans être montrée au spectateur, même pendant l'entracte :

[Le poète] doit bien prendre garde de tomber dans une faute très grossière, [...] qui est de supposer dans l'intervalle d'un acte une chose qui ne peut vraisemblablement avoir été faite sans être vue ; ce qui arrive quand on suppose qu'elle a été faite dans le lieu de la scène : car étant ouvert et exposé aux yeux des spectateurs, ils doivent vraisemblablement avoir vu tout ce qui s'y passe, ou bien il n'est pas vraisemblable que cette chose y soit arrivée, puisqu'ils ne l'y ont pas vue ${ }^{11}$. (D'Aubignac [1657] $2001: 353)$

Même si les personnages sortent, il faut donc considérer que la "représentation " continue : représentation d'un lieu vide, mais qui relève du même régime mimétique que n'importe quelle autre scène. Il s'agit là d'une fiction purement théorique (puisque le spectateur de théâtre quitte bien, quant à lui, sa position de spectateur à l'entracte, et détourne son attention du plateau), mais qui est révélatrice de la manière dont le $\mathrm{XVII}^{\mathrm{e}}$ siècle pouvait valoriser la continuité du spectacle et éprouvait une certaine réticence à envisager sa segmentation.

L'impossibilité d'interrompre le spectacle ailleurs qu'après la sortie des personnages interdit donc nécessairement de créer un suspense « court ». S'y ajoute évidemment la préférence du théâtre classique pour les scènes dialoguées et la rareté des scènes d'action. Mais dans ce système de découpage, même une scène d'action ne pourrait faire l'objet d'un traitement comparable à celui du combat de Don Quichotte et du Biscayen. Par ailleurs, l'exemple d'Échenoz que nous citions plus haut montre bien que 
l'effet de rupture suscité par la coupure interchapitrale ne se réduit pas au cliffhanger à proprement parler, et peut s'appliquer à des scènes dialoguées.

La possibilité de baisser un rideau qui masque le plateau alors que les acteurs s'y trouvent encore (ou, ce qui revient au même, de faire le noir) change radicalement l'effet que peut produire l'entracte. Dans ce cas, c'est le rideau et non plus la sortie des personnages qui marque le passage d'un acte à un autre, si bien que l'interruption du spectacle peut se faire en plein milieu d'une action. Cette intervention d'une instance extérieure à la diégèse (le narrateur qui s'arrête, le rideau qui tombe, le noir qui se fait) est nécessaire pour créer chez le spectateur ou le lecteur cette sensation de manque qui est propre à l'effet de rupture : en effet, cette frustration provient du fait que quelque chose nous cache momentanément une partie de la diégèse; et ce rôle ne peut être tenu que par une instance qui se situe à l'extérieur des événements racontés ou représentés, et non par les événements eux-mêmes, car il est impossible de se cacher derrière soi-même.

Le rideau est devenu une métonymie du théâtre, tant nous y sommes habitués. Son apparition est pourtant relativement récente, et plusieurs études ont montré que «le rideau est $\mathrm{au} \mathrm{XVI}^{\mathrm{e}}$ et $\mathrm{au} \mathrm{XVII}^{\mathrm{e}}$ siècle un élément du spectacle qui reste exceptionnel » (Scherer 1950 : 171). Certaines salles en installent un, notamment pour les spectacles à machines ou le théâtre en musique, mais il n'est dans tous les cas jamais utilisé entre les actes (Scherer $1950: 173^{12}$ ) : « Le rideau, quand rideau il y a, ne vient point cacher la scène pendant les entractes. C'est un assez grand effort, lorsqu'on dispose d'un rideau, de le faire disparaître quand la pièce commence. On ne se soucie pas de répéter une manœuvre si laborieuse. »

Cette absence de rideau à l'entracte est notamment prouvée par le passage de d'Aubignac que nous citions plus haut, et qui ne peut se comprendre que si le plateau reste en permanence à la vue des spectateurs. Marmontel déplore également qu'on ne l'utilise pas systématiquement, ce qui montre que tel n'était toujours pas le cas au XVIII ${ }^{\mathrm{e}}$ siècle (Marmontel [1787] 2005: 489) : "Ne fût-ce même que pour cacher le besoin qu'on a quelquefois de baisser la toile, il serait à souhaiter qu'on la baissât toujours dès qu'un acte serait fini. »

$31 \mathrm{Au} \mathrm{XIX}{ }^{e}$ et $\mathrm{au} \mathrm{xx}^{\mathrm{e}}$ siècles, en revanche, les dramaturges exploitent pleinement les potentialités du rideau d'avant-scène. Cette évolution est fortement liée à la pratique du changement de décor à l'entracte (c'est le «besoin » qu'évoquait Marmontel), et il serait intéressant d'étudier en détail comment, premièrement, s'est opérée la transition entre changement de décor à vue (pratiqué au XVII ${ }^{e}$ siècle dans les pièces à machines) et changement de décor masqué par le rideau ; deuxièmement, à partir de quand le rideau a été utilisé même en l'absence de changement de décor ; troisièmement, comment a évolué, qu'il y ait ou non changement de décor, le rapport entre la sortie des personnages et le rideau : le rideau tombe-t-il après la sortie des personnages (sortie qui contribue donc toujours à affaiblir l'impression de discontinuité) ou bien ceux-ci sont-ils encore présents sur le plateau ${ }^{13}$ ? On se limitera ici à quelques exemples illustrant ce dernier cas de figure, le seul, comme on l'a vu, qui soit susceptible de susciter un véritable effet de rupture au théâtre.

Le quatrième entracte de Le Roi s'amuse est le premier exemple attesté d'un tel procédé chez Hugo. Triboulet a attiré le roi dans la cabane de Saltabadil et a payé ce dernier pour le tuer, sans lui révéler son identité. Maguelonne, la sœur de Saltabadil, tombe sous le charme de l'inconnu, qui dort désormais dans le grenier sans se douter du 
danger qui le menace. Le frère et la sœur décident donc de tuer à sa place la première personne qui frappera à leur porte. De son côté, la fille de Triboulet, Blanche, est amoureuse du roi (sans connaître non plus son identité). Elle assiste à la scène, cachée à l'extérieur de la cabane (la scénographie permet de voir l'intérieur comme l'extérieur). Afin de sauver son amant, elle se sacrifie en entrant en connaissance de cause chez Saltabadil :

BLANCHE, avec horreur, à part

La sœur aide le frère.

Ô Dieu ! pardonnez-leur ! - Pardonnez-moi, mon père !

Elle entre. Au moment où elle paraît sur le seuil de la cabane, on voit Saltabadil lever son poignard. La toile tombe. (Hugo $1832:$ IV, 5)

On voit à quel point cette fin d'acte diffère du modèle classique: la représentation s'interrompt sur un geste inachevé, au moment où la tension est la plus forte. Loin que l'entracte soit précédé par une sortie des personnages et un effet de cadence mineure, il coïncide avec l'instant de la rencontre.

Victor Hugo réemploie un procédé tout à fait comparable dans Lucrèce Borgia. Les sbires du duc s'apprêtent à entrer chez Gennaro pour l'arrêter :

\section{PREMIER HOMME}

L'homme sera pendu. Prends-le. Adieu.

DEUXIÈME HOMME

Bonsoir.

L'autre une fois disparu, il ouvre la porte basse sous le balcon, y entre, et revient un moment après accompagné de quatre sbires avec lesquels il va frapper à la porte de la maison où est entré Gennaro. La toile tombe. (Hugo 1833 : I, deuxième partie, 4)

Là encore, l'acte se clôt sur une action interrompue en plein cours : on devine la suite, mais le moment précis de l'arrestation nous est dérobé, ce qui suscite ce sentiment de manque caractéristique des fins suspensives.

Comme la coupure interchapitrale, le rideau peut également tomber sur la révélation d'un secret. Il accentue ainsi l'effet de surprise. Dans Le Diable et le bon Dieu, Gœtz, qui assiège la ville de Worms, lance un défi à Dieu et joue aux dés pour savoir s'il détruira la ville, ou s'il se consacrera à faire le bien. C'est la seconde solution que désigne le sort :

\section{GCETZ}

L'aube et le Bien sont entrés sous ma tente et nous ne sommes pas plus gais : celle-ci sanglote, celui-ci me hait: on se croirait au lendemain d'une catastrophe. Peut-être que le Bien est désespérant... Peu m'importe, d'ailleurs, je n'ai pas à le juger, mais à le faire. Adieu. Il sort.

Catherine éclate de rire.

CATHERINE, riant aux larmes

Il a triché ! Je l'ai vu, je l'ai vu, il a triché pour perdre.

Rideau. (Sartre $1951: \mathrm{I}$, troisième tableau, 6)

Juste avant l'entracte, le spectateur est laissé brutalement à lui-même, après une réplique qui le force à réinterpréter l'épisode qui vient d'avoir lieu.

Le rideau qui tombe à la fin du premier entracte d'En attendant Godot est un cas particulier qui mérite également de retenir notre attention. Contrairement aux exemples précédents, il ne sert pas tant à créer un effet de rupture en interrompant une action en cours qu'à souligner l'immobilité des personnages.

\section{ESTRAGON}

Alors, on y va?

VLADIMIR

Allons-y. 
Ils ne bougent pas.

Rideau. (Beckett $1952:$ I)

Comme dans le modèle classique, l'acte se termine sur un " allons ", mais cette formule est ici contredite par l'attitude statique de Vladimir et Estragon. Or cette antithèse ne serait pas possible sans rideau (ou, encore une fois, sans un dispositif comparable, comme le noir). L'entracte d'En attendant Godot est donc un hapax : il n'accentue pas ce qui précède, mais y apporte un démenti, il ne fonctionne pas comme une rétention d'information, mais participe pleinement à la création du sens.

Le dispositif dramatique diffère à plusieurs égards du dispositif romanesque. Néanmoins, nous avons vu que les deux genres étaient parcourus par un même débat théorique, dont il conviendrait évidemment d'approfondir la contextualisation dans des travaux ultérieurs, afin de mettre en lumière les enjeux spécifiques des formes qu'il a pu prendre selon les genres, les auteurs et les périodes. Par ailleurs, alors que la coupure interchapitrale (qui suppose une intervention du narrateur) et le modèle de l'entracte classique (marqué, au sein de la diégèse, par la sortie des personnages) sont totalement incompatibles l'un avec l'autre, l'apparition du rideau de scène permet aux dramaturges d'utiliser des procédés qui ne sont pas sans rappeler ceux mis en œuvre par les romanciers en fin de chapitre, même si cette analogie rapide mériterait d'être examinée plus en détail, en prenant en compte l'évolution complexe qu'a connue le théâtre du XVIII ${ }^{\mathrm{e}}$ siècle à nos jours.

Randa Sabry (1992: 129) a bien insisté la prégnance du «mythe linéaire» et du « rectiligne » au XVII ${ }^{e}$ siècle. Comme l'a montré Ugo Dionne, cette linéarité se traduit à la fois par une réticence à l'égard de la segmentation, à laquelle on préfère le modèle de la prose «filée» (Dionne 2008: 360), et un usage de la segmentation (quand segmentation il y a) qui ne cherche pas particulièrement à susciter d'effet de rupture (Dionne 2008: 526): "L'Ancien Régime ne réalise que fort progressivement les potentialités suspensives du blanc interchapitral. » L'exemple de Don Quichotte est à cet égard une irrégularité qui s'explique par le caractère parodique et burlesque du texte. Le dispositif romanesque a donc connu une profonde évolution (Dionne 2008:360): "Cette linéarité, que le classicisme associait diversement à la vérité, à la lisibilité, à la transparence et à l'intelligibilité, ne fera plus partie des pratiques habituelles du XIx ${ }^{e}$ siècle.» Et il est frappant de constater que, mutatis mutandis, le dispositif théâtral a connu une évolution comparable: l'utilisation du rideau à l'entracte permettant d'introduire des effets de discontinuité qui était impensables auparavant. En ce sens, l'évolution conjointe des deux genres correspond également à une convergence de leurs modèles de structuration respectifs.

BIBLIOGRAPHIE 


\section{Sources primaires}

Baudoin, Jean, 1631, Histoire negre-pontique, Paris, Dubray.

Beckett, Samuel, 1952, En attendant Godot, Paris, Minuit.

Camus, Jean-Pierre, 1622, Agathonphile ou les Martyrs siciliens, Paris, Claude Chappelet.

Cervantès, Miguel de, [1605] 2001, L'Ingénieux Hidalgo Don Quichotte de la Manche, éd. et trad. Jean Canavaggio, Paris, Gallimard, coll. « Bibliothèque de la Pléiade ».

Clément, Jean-Marie, 1784, De la tragédie, pour servir de suite aux lettres à Voltaire. Seconde partie, Amsterdam, Paris, Moutard.

Corneille, Pierre, [1660] 1999, « Discours des trois unités, d'action, de jour, et de lieu », éd. Marc Escola et Bénédicte Louvat-Molozay, dans Trois discours sur le poème dramatique, Paris,

Flammarion, coll. « GF ».

Corneille, Pierre, 1637, Le Cid, Paris, Augustin Courbé.

Corneille, Pierre, 1647, Rodogune, princesse des Parthes, Paris, Toussaint Quinet.

D’Aubignac, François Hédelin, [1657] 2001, La Pratique du théâtre, éd. Hélène Baby, Paris, Honoré Champion.

Dacier, André, 1692, La Poétique d'Aristote, traduite en français avec des remarques critiques sur tout l'ouvrage, Paris, Claude Barbin.

Donat, [IV siècle] 2009, Aelii Donati quod fertur commentum Terenti, éd. et trad. Bruno Bureau, Christian Nicolas et Emmanuelle Raymond, dans Hyperdonat, [En ligne], http://hyperdonat.humanum.fr/editions/html/DonEun.html, consulté le 2 septembre 2018.

Échenoz, Jean, 1999, Je m'en vais, Paris, Minuit.

Estienne, Charles, [1542] 1949, Première comédie de Térence, intitulée l'Andrie, nouvellement traduite de latin en français, éd. Harold Walter Lawton, dans Handbook of French Renaissance dramatic theory, Manchester, Manchester University Press.

Fénelon, [1699] 1700, Les Aventures de Télémaque, Bruxelles, Foppens.

Fielding, Henry, [1742] 1964, Les Aventures de Joseph Andrews et de son ami M. Abraham Adams, éd. et trad. Francis Ledoux, Gallimard, coll. « Bibliothèque de la Pléiade ».

Galland, Antoine, [1706] 1965, Les Mille et une nuits, t. II, trad. Antoine Galland, Paris, Flammarion, coll. « GF ».

Hugo, Victor, 1832, Le Roi s'amuse, Paris, E. Renduel.

Hugo, Victor, 1833, Lucrèce Borgia, Paris, E. Renduel.

Marmontel, Jean-François, [1787] 2005, Éléments de littérature, éd. Sophie Le Méhanèze, Paris, Desjonquères.

Sartre, Jean-Paul, 1951, Le Diable et le bon Dieu, Paris, Gallimard.

\section{Textes critiques}

Baroni, Raphaël, 2007, La Tension narrative. Suspense, curiosité et suprise, Paris, Seuil. 
Brecht, Bertolt, [1949] 2000, « Petit organon pour le théâtre ", éd. Jean-Marie Valentin, trad. Bernard Lortholay, dans Écrits sur le théâtre, Paris, Gallimard, coll. « Bibliothèque de la Pléiade ».

Conrad, Clinton C., 1915, The Technique of Continuous Action in Roman Comedy, Menasha, The Collegiate Press.

Dionne, Ugo, 2008, La Voie aux chapitres. Poétique de la disposition romanesque, Paris, Seuil.

Douguet, Marc, 2015, La Composition dramatique. La liaison des scènes dans le théâtre français du XVII siècle, thèse de doctorat sous la direction de Marc Escola, Université Paris 8.

Dupont, Florence et Letessier, Pierre, 2012, Le Théâtre romain, Paris, Armand Colin.

Hamon, Philippe, 1975, « Clausules », Poétique, n² 24, p. 495-526.

Hamon, Philippe, 2017, « Ouverture », dans C. Colin, T. Conrad et A. Leblond (dir.), Pratiques et poétiques du chapitre du XIXe au XXI e siècle, Rennes, PUR.

Iser, Wolfgang, [1973] 1985, L'Acte de lecture. Théorie de l'effet esthétique, trad. Évelyne Sznycer, Bruxelles, Pierre Mardaga.

Leblond, Aude, 2012, « Le chapitre invisible? ", dans Chapitres. Pratique et poétique du chapitre aux XIX et XX $X^{e}$ siècles, [En ligne], https://chapitres.hypotheses.org/62, consulté le 2 septembre 2018.

Lyons, John D., 1999, Kingdom of Disorder. The Theory of Tragedy in Classical France, West Lafayette, Purdue University Press.

Nicolas, Christian, 2008, « À la recherche des fins d'acte et des fins de scène dans les comédies de Térence lues par Donat », dans B. Bureau et C. Nicolas (dir.), Commencer et finir. Débuts et fins dans les littératures grecque, latine et néo-latine, t. II, Lyon, Université Jean Moulin - Lyon 3, p. 595-620.

Pasquier, Pierre, et Surgers, Anne (dir.), 2011, La Représentation théâtrale en France au XVII siècle, Paris, Armand Colin.

Sabry, Randa, 1992, Stratégies discursives. Digression, transition, suspens, Paris, Éditions de l'EHESS.

Scherer, Jacques, 1950, La Dramaturgie classique en France, Paris, Nizet.

Selmeci Castioni, Barbara, 2011, « Poétique du continu et usages de la séquence. Le Grand Cyrus à la croisée de différentes pratiques d'écriture, de lecture et d'édition (XVII ${ }^{\mathrm{e}} \mathrm{XXI}{ }^{\mathrm{e}}$ siècles) », dans S. Triaire et P. Victorin (dir.), Deviser, diviser. Pratiques du découpage et poétiques du chapitre de l'Antiquité à nos jours, Montpellier, PULM, p. 173-174.

Védier, Georges, 1955, Origine et évolution de la dramaturgie néo-classique. L'influence des arts plastiques en Italie et en France : le rideau, la mise en scène et les trois unités, Paris, PUF.

\section{NOTES}

1. On rapprochera cette position de celle d'Antoine Galland, qui se justifie d'avoir supprimé la division en nuits dans l'avertissement au tome VII de sa traduction des Mille et une nuits (Galland [1706] 1965 : 257). Sur ce texte, voir la contribution de Jérémy Naïm dans ce numéro.

2. "Praefatio", en tête de Donat, "Aelii Donati in Eunuchum Terenti commentum ", dans Donat ([IV siècle] 2009). Dans la comédie latine on lève (ou dresse) un rideau qui 
cache le plateau à la fin de la pièce. Pour une contextualisation historique de ce genre, voir notamment Dupont et Letessier (2012).

3. «J'ai donc premièrement considéré l'ordre de l'ouvrage, que j'ai divisé par livres et par chapitres, pour le soulagement de ceux qui prendront la peine de le lire » (Baudoin 1631 : n. p., cité par Dionne $2008: 254)$; « On a trouvé à propos de diviser cet ouvrage en seize livres, pour reposer le lecteur " (Fénelon [1699] $1700:$ n. p., cité par Dionne 2008 : 222).

4. Nous soulignons.

5. Nous soulignons.

6. Sur ces catégories, voir Baroni (2007).

7. Les séries télévisées fourniraient évidemment bien d'autres exemples intéressants de ce procédé, le montage remplissant la fonction de segmentation dévolue au narrateur.

8. Sur ces deux modèles de composition, voir Scherer (1950:266-284). Voir également Douguet (2015).

9. Donat, «Aelii Donati in Andriam Terenti commentum », II, 3, dans Donat ([IV siècle] 2009).

10. Voir notamment Conrad (1915) et Nicolas (2008).

11. Sur ce passage, voir notamment l'analyse de John D. Lyons (1999: 186).

12. Voir également Védier (1955).

13. Sur ce sujet, outre Scherer (1950) et Védier (1955), on pourra se reporter notamment à Pasquier et Surgers (2011).

\section{RÉSUMÉS}

Dans un roman, la coupure interchapitrale permet d'accroître la tension narrative en interrompant le récit d'une action avant son terme. La division en actes d'une pièce de théâtre a connu quant à elle une évolution importante : à l'âge classique, la fin d'un acte correspond nécessairement à la sortie des personnages, qui laissent le plateau vide; ce n'est que plus tard que le rideau de scène sera utilisé pour marquer cette coupure. Afin de comprendre les enjeux de cette spécificité, cet article cherche à comparer d'une part les effets que peuvent produire, dans les différentes formes qu'ils ont prises, le dispositif théâtral et le dispositif romanesque et, d'autre part, les débats théoriques qu'ils ont chacun suscités.

In a novel, interchapters can increase the narrative tension by interrupting the narration of an action before it ends. In the dramatic genre, act-division has evolved: during the classical age, all characters exit at the end of an act, thus leaving the stage empty; only later will the curtain be used to underline this break. This paper aims at understanding what this specifity involves and at compairing on the one hand the effect created by the different forms of narrative and dramatic structuration systems, and, on the other hand, the theoretical debates they raised. 
INDEX

Mots-clés : actes, chapitre, théâtre, suspense, rideau

Keywords : acts, chapter, drama, suspense, curtain

\section{AUTEUR}

\section{MARC DOUGUET}

Université Grenoble Alpes, UMR Litt\&Arts 